\title{
Limb Body Wall Complex: A Rare Anomaly
}

\author{
Unan Sultana ${ }^{1 *}$ \\ Md. Qumrul Ahsan
}

'Department of Radiology and Imaging Chattagram Maa-O-Shishu Hospital Medical College Chittagong, Bangladesh.

\section{${ }^{*}$ Correspondence to:}

\section{Dr. Unan Sultana}

Assistant Professor

Department of Radiology and Imaging

Chattagram Maa-O-Shishu Hospital Medical College

Chittagong, Bangladesh.

Mobile : +8801814267331

E-mail: una.areeb@gmail.com

www.banglajol.info/index.php/CMOSHMCJ

\begin{abstract}
Body stalk anomalies are a group of massively disfiguring abdominal wall defects in which the abdominal organs lie outside of the abdominal cavity in a sac of amnioperitoneum with absence of or very small umbilical cord. Various hypotheses proposed to explain the pathogenesis of limb body wall complex include early amnion disruptions, embryonic dysplasia, and vascular disruption in early pregnancy. Body stalk anomaly is an accepted fatal anomaly and, hence, its early diagnosis aids in proper management of the patient. We present a case of LBWC, exhibiting combined cranial, abdominal \& limb features.
\end{abstract}

Key words : Abdominal wall defects; Umbilical cord; Fetal malformation; LBWC.

\section{INTRODUCTION}

Limb Body Wall Complex (LBWC) is a rare complicated sporadic polymalformative fetal malformation syndrome, characterized by a wide spectrum of severe anomalies in the body wall. The incidence at birth is about 0.32 per 100,000 births because the majority of affected fetuses undergo intrauterine deaths ${ }^{1,2,3}$. Traditionally diagnosis has been based on the Van Allen et al criteria, i.e. the presence of two out of three of the following anomalies ${ }^{4}$ :-

i) Exencephaly or encephalocele with facial clefts

ii) Thoraco and or abdominoschisis and

iii) Limb defects.

In our case, we described a live fetus of 28 weeks having anencephaly with parieto occipital mass, gastroschisis and limb defect.

\section{CASE REPORTS}

A 20-year-old primigravida female with a 8-month amenorrhea was referred to Radiology and Imaging Department for routine ultrasonography and fetal well-being study on 07.08.2016. She had normal blood profile and B+ve blood group. Hemoglobin was $10.5 \mathrm{gm} / \mathrm{dL}$. The ultrasonography revealed FL $5.2 \mathrm{~cm}$ corresponding to 28 weeks. Biparietal Diameter (BPD) not measured as there was absence of part of cranial bones. One cystic area, measuring $(5.9 \mathrm{~cm} \times 3.8 \mathrm{~cm})$ was noted in the occipital region. A large abdominal wall defect, with liver gut coils \& both kidneys herniating through it, into the liquor amnii without covering membrane was seen. Fetal spines appear normal (Figure 1A,B). During further scan one of the upper limb could not visualized. No anomaly was detected in the eyes, palate, lips, face, and thoracic region. The female was referred back to Department of Gynecology and Obstetrics for further management with diagnosis of LBWC. On counseling, the patient about the fatal outcome of the anomaly, the pregnancy was terminated on 09.08.2016 , a male baby was delivered by SVD and ultrasonography findings confirmed thereof.

Examination of the fetus revealed a large gastroschisis with herniation of the small intestine and liver. Left upper limb was absent, but right upper \& both lower limbs were normal. Fetus also had anencephaly with large mass at parieto occipital region (Figure 2). 


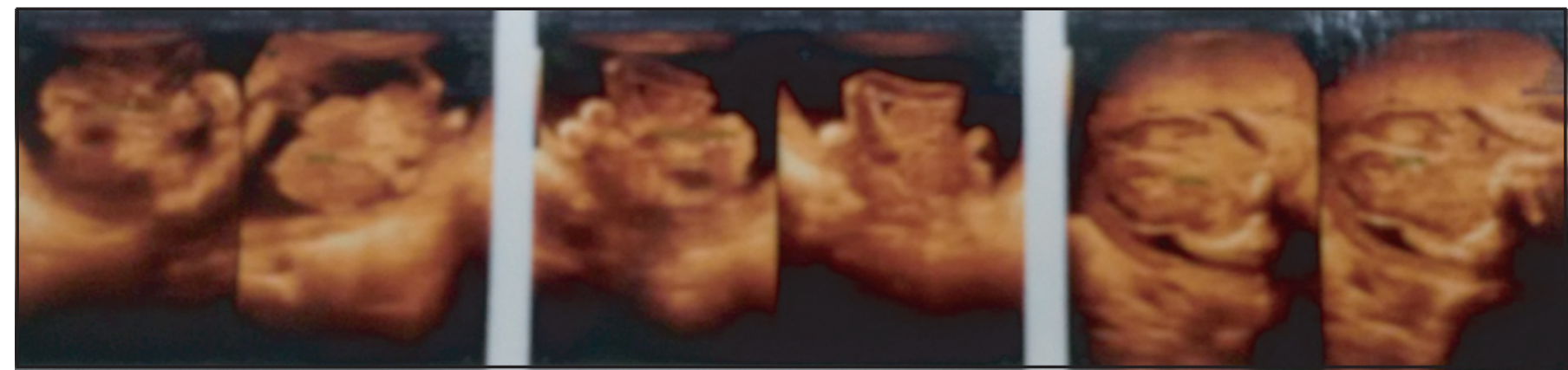

Figure $1 \mathrm{~A}$ : Ultrasound image showing the large abdominal wall defect with liver and gut-coils herniating through it into the liquor amnii.

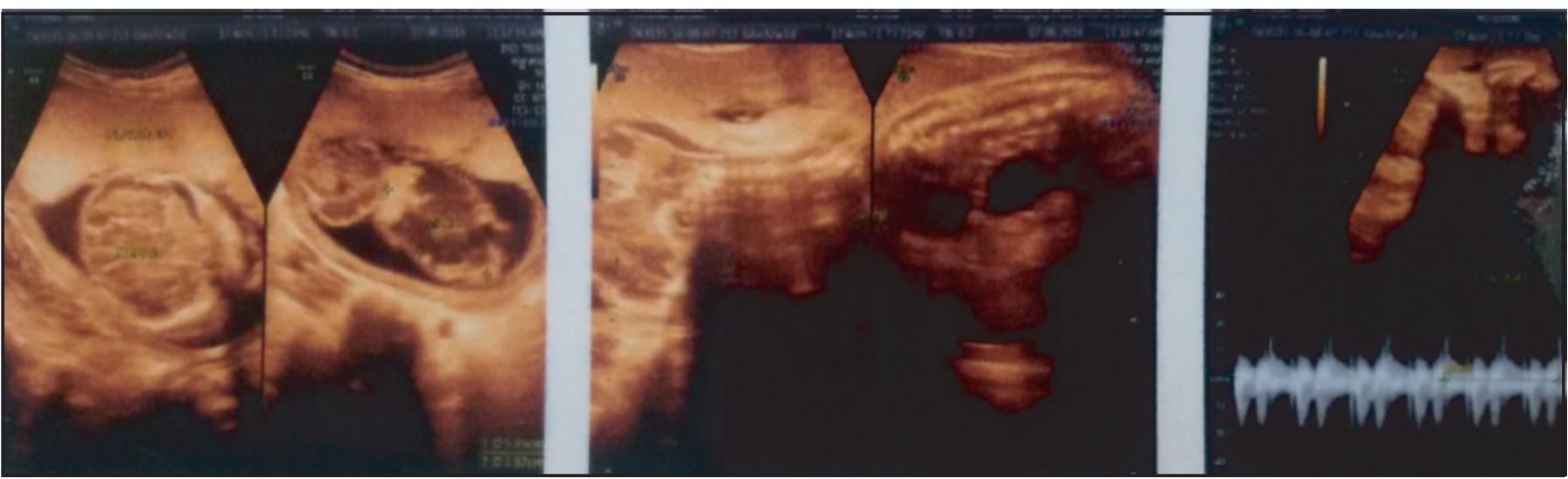

Figure 1 B : Mass at occipital region.

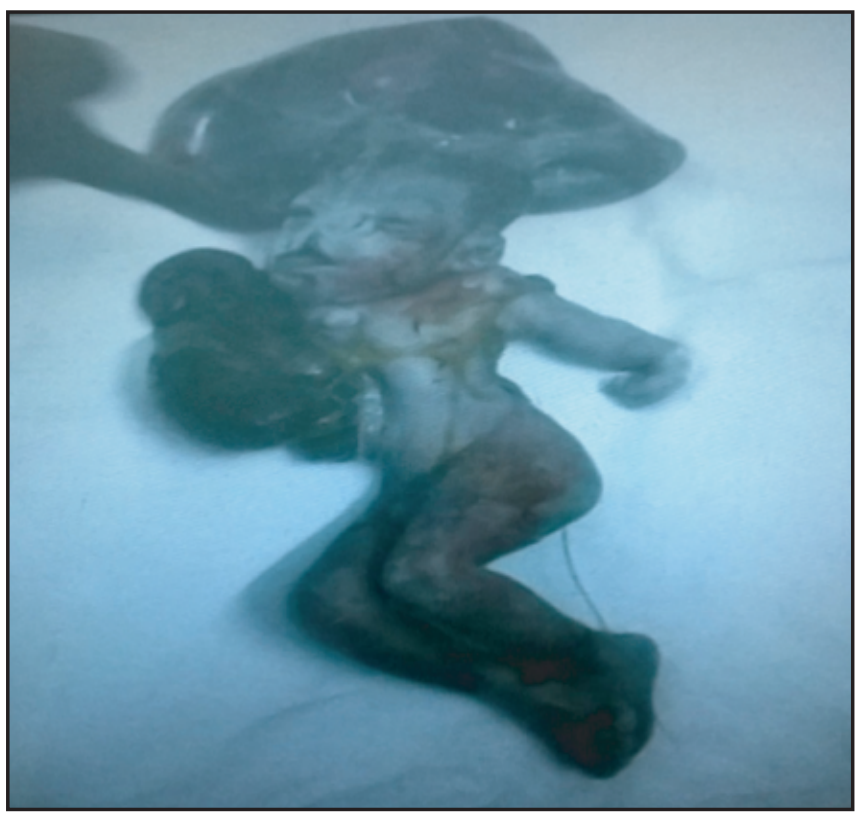

Figure 2 : Fetus with large mass at parieto occipital region.

\section{DISCUSSION}

LBWC was described for the first time by Van Allen et al in $1987^{4}$. It is also known by the other names like "Body stalk anomaly" "Congenital absence of umbilical cord" and "cyllosomus and Pleurosomus" 3,5 . The diagnostic criteria for LBWC is still debatable, the commonly used one was put forth by Van Allen et al in1987 as stated earlier. Russo et al in 1993 identified two distinct phenotypes Type I (Craniofacial defects, facial clefts, amniotic adhesions and amniotic band syndrome) and Type II (No craniofacial defects, Urogenital anomalies, imperforate anus, lumbosacral meningomyelocele, severe kyphoscolosis and placental anomalies) ${ }^{6}$. The sole criteria used by Russo et al was craniofacial defects in Type I and many other thorasic and abdominal anomalies in Type $\mathrm{II}^{6}$. To overcome this deficiency Sahinoglu et al in 2007 proposed a new classification: ${ }^{7}$

Type I : (Fetus has craniofacial defect and intact thoracoabdominal wall, often normal placenta and umbilical cord but rarely attached to the malformated cranial structures)

Type II : (Fetus has supraumbilical, usually laterally located (Often left side) large thoracoabdominal wall defect. Eventrated abdominal organs enveloped within the amniotic sheet connects to the skin margin of the wall defect. No well-formed umbilical cord and usually normal cloacal structures are detected)

Type III : (Fetus has infraumbilical abdominal wall defect with intact thorax. The placenta is attached broadly to the skin at the site of defect. The abdominal organs are eventrated into the extraembryonic coelomic cavity. The cloacal structures are almost always malformed or absent). 
Our case obeyed Van Allen criteria and was distinct from the fetuses observed by Russo et al and Sahinoglu et $\mathrm{al}^{6,7}$.

The exact etiology of this condition is still unclear, Tropin's amniotic band theory and Van Allen's vascular theory failed to explain all the anomalies observed in $\mathrm{LBWC}^{4,8}$. The most accepted theory is early embryonal dysplasia put forward by Hartwig et al in $1989^{\circ}$. According to this, there will be an abnormal embryonic folding related to malfunctioning of the body wall ectodermal placode. This leads to defective closure of embryonic abdominal wall, umbilical abnormality and persistence of extra embryonic coelom communicating with the abdominal cavity. These ectodermal placodes also add cells to mesoderm of the trilaminar embryonic disc, which is destined to form the genitourinary tract. Some authors suggest that vascular disruption is secondary to hypoplasia of the blood vessel in the affected area rather than being the primary etiological factor ${ }^{7}$. This anomaly does not have any sex predilection and recurrence of this condition is observed in two families suggesting a possible genetic etiology ${ }^{10}$.

LBWC is a heterogeneous disease and is associated with varied internal anomalies. The central nervous system anomalies observed are, anencephaly, encephalocele and alobar holoprosencephaly. Cardiovascular anomalies include primitive ventricle, common atrium, atrial septal defects, truncus arteriosus, membranous Ventricular septal defects hypoplastic right ventricle and ectopic cardis. The renal anomalies observed are unilateral or bilateral aplasia/hypoplasia of kidney, hydronephrosis, renal dysplasia, polycystic kidney and calcification of kidney. Genital abnormalities seen are abnormal external genitalia, absent gonad and extrophy of bladder. Skeletal anomalies are most common and include club foot, oligodactyly, arthrogryposis, absent limb, single forearm bone, single lower leg bone, pesudosyndactyly, radial/ulnar hypoplasia, rotational defects and polydactyly. Other anomalies include trilobulated liver, polysplenia, absent gall bladder, amniotic bands and single umbilical artery. We have observed complex cranial and abdominal anomalies which are not observed in the literature ${ }^{3,5,11}$.

\section{CONCLUSION}

Prenatal ultrasound examination (USG) can detect this anomaly as early as first trimester. The USG findings of this anomaly are the presence of abdominal wall defects and limb abnormality. LBWC is a lethal anomaly hence it has to be differentiated from treatable causes like omphalocele or gastroschisis, but sometimes oligohydramnios may mask the underlying anomalies and therefore, challenge the radiologist. Early diagnosis followed by medical termination is the preferred treatment for this anomaly ${ }^{3}$.

So ultrasonographic protocol for obstetrics should be followed in every obstetric case and thus early detectection of congenital anomaly and treatment can be possible. Thus we can reduce delivery rate of abnormal babies. Which is beneficial for the parents, society and the country.

\section{DISCLOSURE}

Both the author declared no competing interest.

\section{REFERENCES}

1. D'souza J, Indrajit IK, Menon S. Limb body wall complex. Med J Armed Forces India. 2004;60:77-80.

2. Prasun P, Behera BK, Pradhan M. Limb body wall complex. Indian J Pathol Microbiol. 2008;51:255-256.

3. Borade A, Prabhu AS, Prabhu GS, Prabhu SR. Limb Body Wall Complex (LBWC) [Last cited on 2009 Jul 1]. Pediatric Oncall. 20096 Art \# 37.

4. Van Allen MI, Curry C, Gallagher L. Limb body wall complex: I. Pathogenesis. Am J Med Genet.1987;28:529-548

5. Managoli S, Chaturvedi P, Vilhekar KY, Gagane N. Limb body wall complex. Indian Pediatr.2003;40:891-894.

6. Russo R, D’Armiento M, Angrisani P, Vecchione R. Limb body wall complex: A critical review and a nosological proposal. Am J Med Genet. 1993;47:893-900.

7. Sahinoglu Z, Uludogan M, Arik H, Aydin A, Kucukbas M, Bilgic R, et al. Prenatal ultrasonographical features of limb body wall complex: A review of etiopathogenesis and a new classification. Fetal Pediatr Pathol. 2007;26:135-151.

8. Torpin R. Amniochorionic mesoblastic fibrous strings and amnionic bands: Associated constricting fetal malformations or fetal death. Am J Obstet Gynecol. 1965;91:65-75.

9. Hartwig NG, Vermeij-Keers C, De Vries HE, Kagie M, Kragt H. Limb body wall malformation complex: An embryologic etiology? Hum Pathol. 1989;20:1071-1077.

10. Plakkal N, John J, Jacob SE, Chithira J, Sampath S. Limb body wall complex in a still born fetus: A case report. Cases J. 2008;1:86.

11. Socolov D, Terinte C, Gorduza V, Socolov R, Puiu JM. Limb body wall complex-case presentation and literature review. Rom J Leg Med. 2009;17:133-138. 